\title{
Integrated in Silico Docking and MOMA Simulation Methods Reveal Rottlerin as a Potent Janus kinase 2 (JAK2) Inhibitor
}

\author{
Jyothirmayi Vadapalli ${ }^{1}$, Anuradha Vanam ${ }^{2}$, Noboru Motohashi ${ }^{3}$ and Rao Gollapudi ${ }^{4}$ \\ ${ }^{1}$ Acharya Nagarjuna University, Nagarjunanagar, India
}

${ }^{2}$ Sri Venkateswara University, India

${ }^{3}$ Meiji Pharmaceutical University, Japan

${ }^{4}$ University of Kansas, Lawrence, USA

Received: 制: October 29, 2018; Published: 制: November 13, 2018

*Corresponding author: Rao Gollapudi, University of Kansas, Lawrence USA

\begin{abstract}
Janus kinase 2 (JAK2) is a prospective drug target for myeloproliferative neoplasms, mainly polycythemia Vera, essential thrombocythemia and primary myelofibrosis disorders. JAK2 abnormalities are mainly identified in myeloid neoplasms. Multiple type I ATP-competitive JAK inhibitors with various specificities displayed potent anti-inflammatory activity with minimum hematologic toxicity in clinical trials. Therefore, only two tyrosine kinase inhibitors have been approved by Food and Drug Administration (FDA) as follows: first tyrosine kinase inhibitor is JAK2/JAK1 inhibitor (ruxolitinib 1) in intermediate-2 and high-risk myelofibrosis and hydroxyurea-resistant or -intolerant polycythemia Vera. Second tyrosine kinase inhibitor is JAK1/JAK3 inhibitor (tofacitinib. 2) in methotrexate-resistant rheumatoid arthritis. However, the potent-antiinflammatory effects of JAK inhibitors seem to be logical therapeutic approach for various inflammatory and auto-immune diseases. Extensive research has identified the chemotherauperic potential of plant-derived compounds of which rottlerin (5) exhibited great potentiality since rottlerin (5) influences various cell mechanisms involved in cell survival, autophagy, apoptosis and invasion. Rottlerin (5) activity is presumed to result from a combination of signaling pathways at multiple levels. Bioinformatics' tools were utilized to identify the specific targets for different ligands. In silico predictions, modeling and dynamic simulations based on the crystal structure of JAK2 and ligands were performed. Active molecular docking studies using AutoDock Vina software suggested that rottlerin (5) is a novel inhibitor of JAK2 with binding affinities of $-9.3 \mathrm{kcal} / \mathrm{mol}$ when compared to approved drugs ruxolitinib (1) $(-8.7 \mathrm{cal} / \mathrm{mol}$ and tofacitinib (2) $(-7.4 \mathrm{kcal} / \mathrm{mol})$. Of these ligands investigated in this study, rottlerin (5) produced the greatest JAK2 binding affinity. The data suggests that rottlerin (5) could be explored as potent lead compound to treat myelofibrosis caused by JAK2 overexpression.
\end{abstract}

Keywords: Janus kinase 2; Myelofibrosis; Docking Studies; In Silico; Apigenin; Gandotinib; Ruxolitinib; Rottlerin; Tofacitinib; Wogonin

Abbreviations: ARG: Argenine; ASN: Aspertine; Glu: Glutathione; Gly: Glycine; LYS: Lysine; PHE: Phenylalanine, VAL: Valine; JAK 2: Janus Kinase 2; ET: Thrombocythemia; MPDs: Myeloproliferative disorders; PV: Polycythemia Vera; PMF: Primary Myelofbrosis

\section{Introduction}

Janus kinase 2 (JAK2) is a non-receptor tyrosine kinase. Janus kinase family (JAK1, JAK2, JAK3 and TYK2) was associated with signaling by members of the type II cytokine receptors (e.g. interferon receptors), gp130 receptors (e.g. IL-6R), GM-CSF receptors (IL-3R, IL-5R and GMCSF-R), and single chain receptors (e.g. Epo-R, Tpo-R, GH-R, PRL-R) [1,2]. JAK/STAT signaling pathway plays imperative part in many physiological processes in response to various extracellular stimuli and cytokines. The Janus kinase family (JAK1, JAK2, JAK3 and TYK2) plays a crucial role in cytokine and growth factor mediated Janus kinase/signal transducers and activators of transcription (JAK/STAT) signal transduction [3]. JAK auto-phosphorylation leads to dimerization of phosphorylated STAT proteins and their translocation into the nucleus, thereby increasing cellular proliferation and resistance to apoptosis [4]. Myelofibrosis is a group of rare cancers of bone marrow and is classified as chronic leukaemia. Among the Janus kinase family, JAK2 was proved to be associated with the pathogenesis of myeloproliferative disorders (MPDs), primarily including polycythemia Vera (PV), essential thrombocythemia (ET), and primary myelofbrosis (PMF) [5-7].

In particular, the essential role in cell growth and survival makes JAK2 an important target for cancer treatment $[8,9]$. However, ruxolitinib (1) was the only JAK1/JAK2 inhibitor that was 
approved for clinical use to treat myelofibrosis and hydroxyurearesistant-moderate- 2 and high risk myelofibrosis and hydroxyurearesistant or intolerant polycythemia Vera. In addition, tofacitinib (2) was approved JAK1/JAK3 inhibitor to treat methotrexateresistant rheumatoid arthritis. JAK inhibitors often exhibit potential anti-inflammatory activity and thus could be useful in chronic inflammation treatment. Therefore, research had been diverted to discover other JAK1/JAK2 inhibitors with better drug-like properties with novel structural skeleton and fewer side effects. Furthermore, non-approved JAK inhibitors displayed numerous offtarget effects leading to undesired neurological and gastrointestinal toxicities in myeloproliferative neoplasm associated clinical trials [10]. Recently, several small molecules belonging to different class of natural products were reported to regulate JAK/STAT pathway [12]. Among these, apigenin (3) and wogonin (4) have been found to inhibit IL-1-induced STAT1/2 activation via inhibition of JAK2 activation, leading to MMP-13 down-regulation in SW1353 cells (human chondrosarcoma cell line) [13].

Rottlerin (5) ((E)-1-[6-[(3-acetyl-2,4,6-trihydroxy-5methylphenyl) methyl]-5,7-dihydroxy-2,2-dimethyl-chromen-8yl]-3-phenyl-prop-2-en-1-one) also known as mallotoxin, is the principal phloroglucinol derivative of kamala dye obtained from Mallotus philippinensis [14]. Rottlerin (5) inhibited many other protein kinases, such as MAPKAPK5 (PRAK), MAPKAP-2, Akt/ $\mathrm{PKB}$, and calmodulin-dependent protein kinase (CaMK) [15]. Rottlerin (5) uncoupled mitochondrial respiration from oxidative phosphorylation, thereby reducing adenosine triphosphate (ATP) levels and affected several cellular functions [13]. In pancreatic acinar cells, rottlerin (5) modulated several biological and biochemical processes in a $\mathrm{PKC} \delta$-independent pathway. In addition, rottlerin (5) (6M) depleted ATP levels, consequently averted the phosphorylation of many signaling proteins and inhibited enzymatic secretion and several intracellular pathways, in a $\mathrm{PKC} \delta$-independent manner. As a result, the inhibitory effects of rottlerin (5) were mimicked by the mitochondrial uncouplers carbonylcyanide m-chlorophenylhydrazone and carbonyl cyanide p-trifluoromethoxy phenylhydrazone, in pancreatic acini [16]. Cancers are caused by deregulation of hundreds of genes. An ideal anticancer agent should target multiple gene products or signaling pathways simultaneously without effecting normal cells. Recently, extensive research was focused on the chemotherapeutic potential of plant-derived compounds. Rottlerin (5) inhibited cell growth, induced apoptosis, arrested cell cycle, and retarder cell invasion as well as migration.

Furthermore, rottlerin (5) suppressed Skp2 expression and subsequently exerted its tumor suppressive function in pancreatic cancer cells, suggesting that rottlerin (5) might be a potential therapeutic target for treating pancreatic cancer [14]. Among the ever-increasing list of naturally occurring anticancer agents, rottlerin (5) was effective in chemotherapy because of its influence on several cellular machineries involved in survival, apoptosis, autophagy, and invasion [17]. In a recent study, rottlerin emerged as the most potent metastasis-associated in colon cancer 1 transcriptional (MACC1) inhibitors [18]. Rottlerin (5) anticancer activity resulted from combination of signaling pathways at multiple levels. Furthermore, the underlying mechanisms described for rottlerin (5) anticarcinogenic activity were diverse and cell-specific [14]. Molecular docking is a computer-based drug design that initiates the docking of a ligand into the active site of the receptor. The motive of receptor and ligand docking interactions can be predicted through three-dimensional visualization of the docked complexes. This research was conducted to explore the binding interactions between receptor protein and the three naturally rottlerin (5) along with JAK known inhibitors, ruxolitinib (1), tofacitinib (2) and gandotinib (6) (Figure 1).<smiles>N#CC[C@@H](C1CCCC1)n1cc(-c2ncnc3[nH]ccc23)cn1</smiles>

Ruxolitinib (1)<smiles>C[C@@H]1CCN(C(=O)CC#N)C[C@H]1N(C)c1ncnc2[nH]ccc12</smiles>

Tofacitinib (2)<smiles>O=c1cc(-c2ccc(O)cc2)oc2cc(O)cc(O)c12</smiles>

Apigenin (3)<smiles>COc1c(O)cc(O)c2c(=O)cc(-c3ccccc3)oc12</smiles>

Wogonin (4)<smiles>[R2]Oc1c(Cc2c(O)c3c(c(C(=O)/C=C/c4ccccc4)c2O)OC(C)(C)C=C3)c(O)c(C)c(O)c1C(C)=O</smiles>

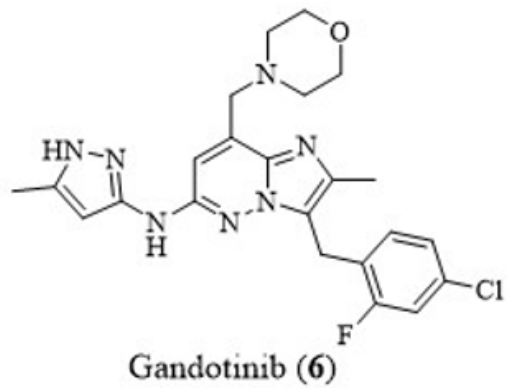

Figure 1: Ruxolitinib (1), Tofacitinib (2), Apigenin (3), Wogonin (4), Rottlerin (5), Gandotinib (6). 


\section{Materials and Methods}

\section{Protein Preparation}

Homology Modeling of the 4AGC Protein: Homology modeling methods make use of experimental protein structures ("templates") to build receptor proteins in drug discovery. Homology (or comparative) modelling is currently the most accurate method to generate reliable three-dimensional protein structure models. SWISS-MODEL is a structural bioinformatics web-server dedicated to homology modelling to predict protein 3D structures [19,20]. Janus kinase 2 (PDB ID: 4AGC), protein structure retrieved from protein data bank (http://www.rcsb.org/) was missing some amino acid residues. The complete JAK2 protein was modelled by submitting FASTA sequence of 4AGC (chain A) protein into SWISS-MODEL Workspace through automated mode for the development of a more accurate protein model [21]. The 4AGC (Chain A) protein and its sequence were selected as the target protein and query sequence, respectively. The crystal structure of JAK2- (PDB ID: 2W1I) was also obtained from the protein data bank. The 4AGC protein model was built by using the $2 \mathrm{~W} 1 \mathrm{I}$ protein as a suitable template. In the SWISS MODEL Workspace automated mode, fifty templates of query sequence were generated. The template, 2W1I.1.A, demonstrated highest sequence identity to query sequence and was used to develop an improved model of the 4AGC protein. Global quality estimate, local quality estimate comparison and 4AGC model template alignment with 2W1I.1.A were calculated (Figure 2) [22,23].

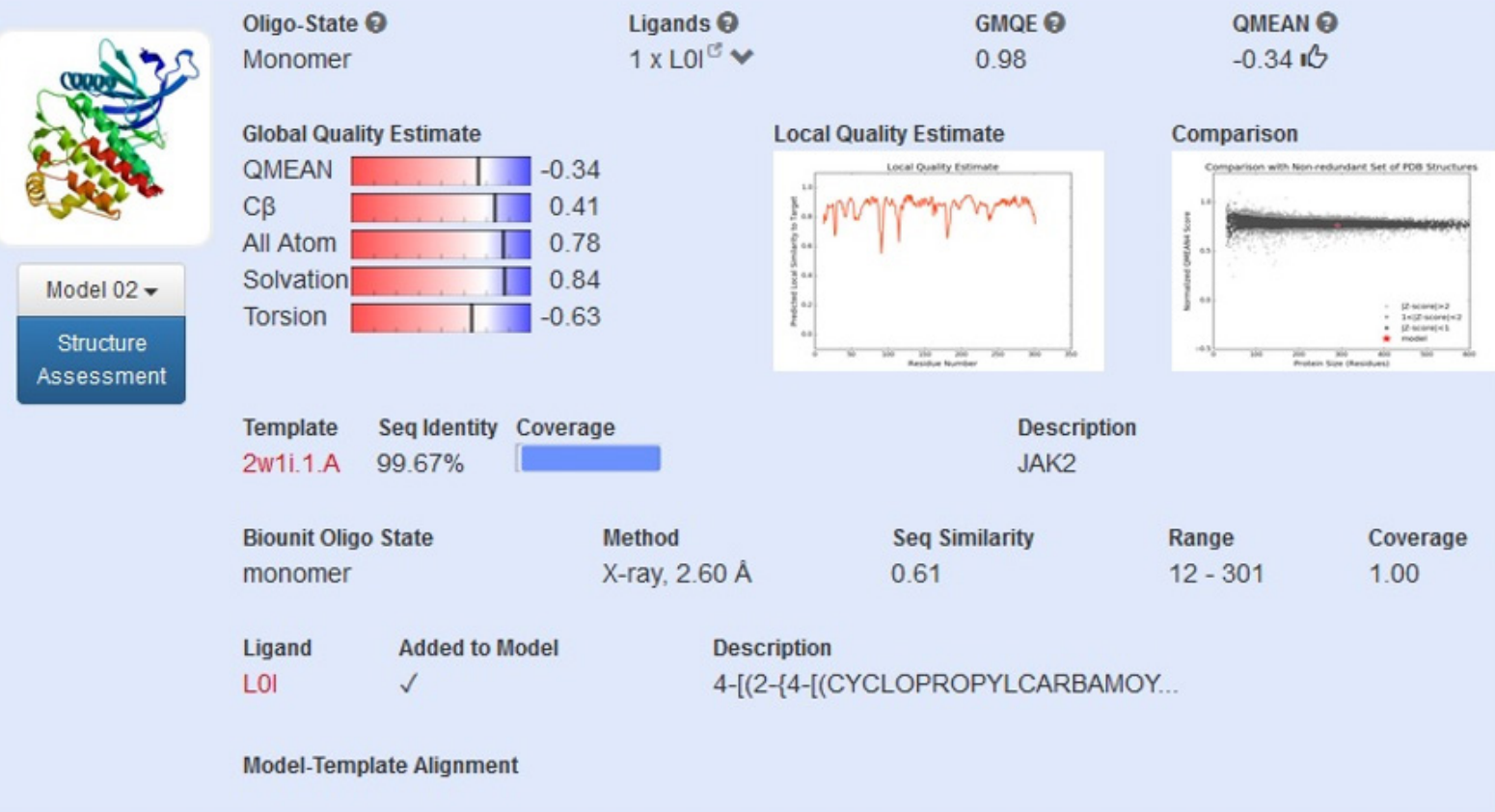

Figure 2: Global quality estimate, local quality estimate comparison and 4AGC model template alignment with 2W1I.1. A.

Modeled JAK2 Protein Validation: The 4AGC-Modeled protein quality was validated by Ramachandran plot using Rampage [24] and in SPDBV (Deep View - Swiss - Pdb Viewer) version 4.10 based on the RMSD value obtained by superimposing the 4AGC protein model on its model template 2W1I [25]. 4AGC-Modeled protein was selected for molecular docking to acquire reliable prediction of ligands ability to bind with the receptor. The quality of 4AGCModeled protein was validated by comparing Ramachandran plots of 4ACC, 2W1I and modeled 4AGC using Rampage [24]. A New generation of crystallographic validation tools for the protein data bank and in SPDBV (Deep View - Swiss - Pdb Viewer) version 4.10 based on RMSD value obtained by superimposing 4AGC protein model on its model [25]. Ramachandran plot values of the 4AGC, 4AGC-Modeled protein and its template 2W1I.1.A were obtained (Table 1). In Ramachandran plot generated for 4AGC protein, $96.8 \%$ of amino acid residues were found in favored region, $2.8 \%$ residues in allowed area, and $0.4 \%$ of residues were present in outlier regions (Figure 3). Ramachandran plot of 2W1I.1.A showed
$94.6 \%$ residues in favored region, $4.0 \%$ residues in allowed region, and $1.4 \%$ of residues in outlier regions. Ramachandran plot generated for 4AGC-Modeled protein displayed 97.2\%, 2.1\% and $0.7 \%$ of residues in the favored, the allowed, and the outlier regions, respectively. Ramachandran plot data for 4AGC-Modelled, 6FTU and 2W1I proteins suggested favorable reliability of 4AGCModelled protein for subsequent docking studies (Table 1) (Figure 3).

Table 1.

\begin{tabular}{|c|c|c|c|}
\hline & \multicolumn{3}{|c|}{ Percent (\%) of Residues Per Region } \\
\hline Structure & Favored & Allowed & Outlier \\
\hline 4AGC-Modeled & 97.2 & 2.1 & 0.7 \\
\hline 4AGC & 96.8 & 2.8 & 0.4 \\
\hline 2W1I & 94.6 & 4.0 & 1.4 \\
\hline
\end{tabular}




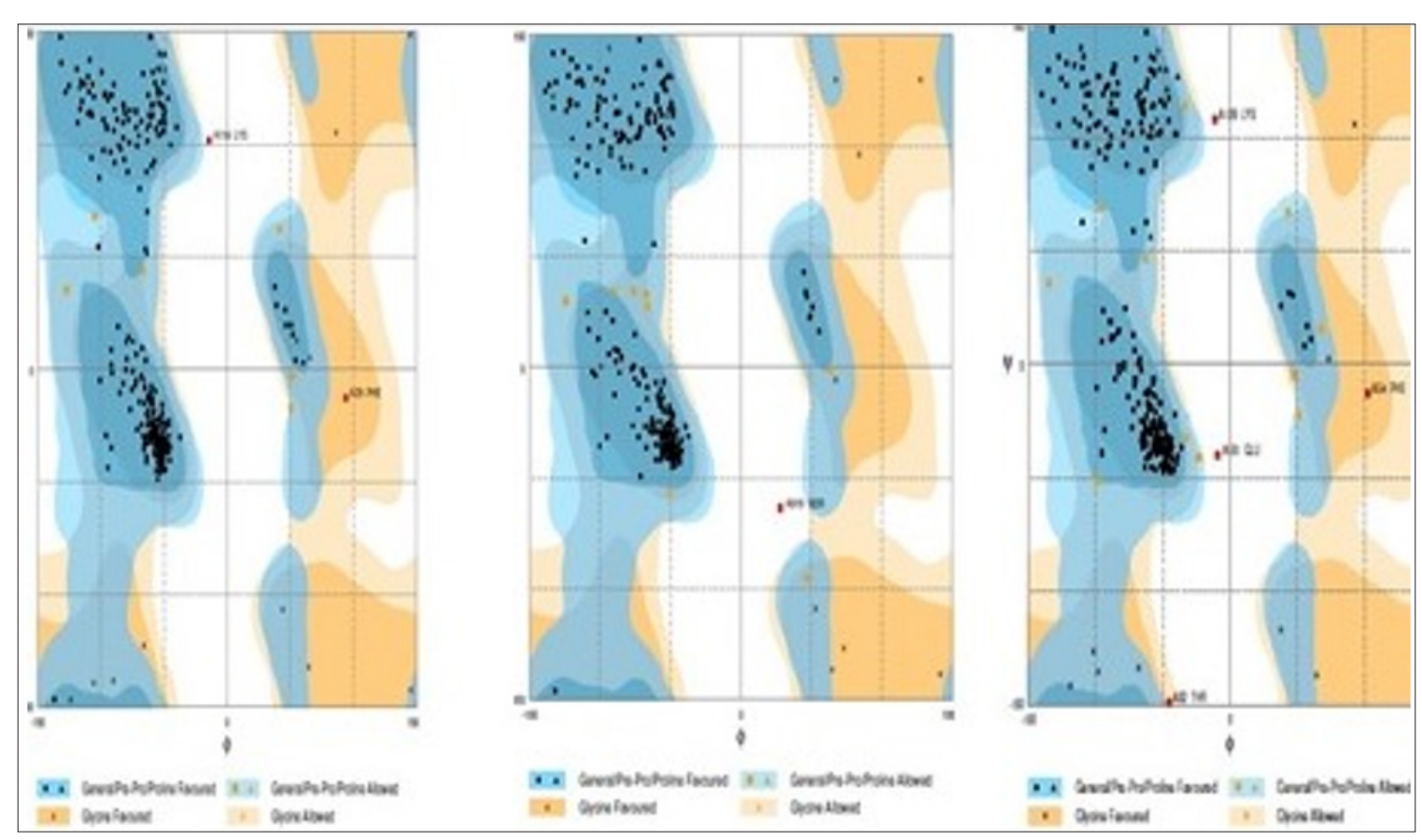

Figure 3: Ramachandran plots for The Model-4AGC (4AGC-Modeled), retrieved 4AGC and 2W1I.

Energy Minimization and Refinement of the Modeled 4AGC Protein (4AGC-Modeled): The modeled 4AGC (4AGC-Modelled) protein valency and chemistry were rectified using Chimera (UCSF, San Francisco, CA, USA). Energy minimization and refinement were performed by employing CHARMm force field in order to obtain a protein with least energy [26,27]. Later, polar hydrogen atoms were added to the protein model using Auto Dock tools 4.2 .6 screening tool and PyRx v0.8 software (http://pyrx.sourceforge.net/).

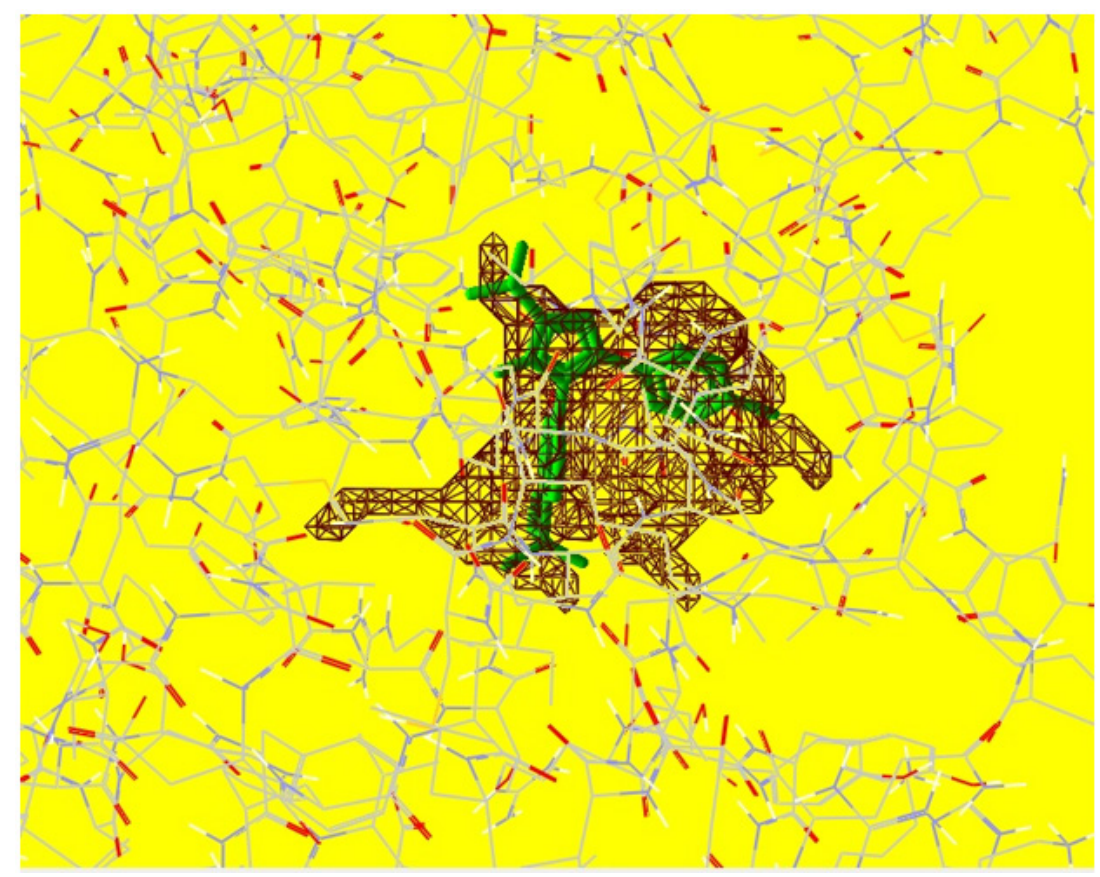

Figure 4: ATP (Green) binding into the active cavity (Brown) of JAK2 kinase.

Receptor Cavity Prediction: The cavity or the potential ligand binding site of JAK2 (PDB ID:4AGC) was predicted using MVD with volume of $174.08 \mathrm{~A} 3$ which was identified as active site for docking using Molegro Virtual Docker (MVD) software. The active binding cavity was further ascertained through a trail docking run of JAK2 and adenosine triphosphate (ATP) (Figure 4).

\section{Preparation of Ligands}

The structures of ruxolitinib (1) (CID: 25126798), tofacitinib (2) (CID: 9926791), rottlerin (5) (CID: 5281847) and gandotinib (6) (CID: 46213929) were initially retrieved from PubChem Compound Database (National Center for Biotechnology Information, U.S. 
National Library of Medicine). Molecular geometry optimization of ligands was achieved using Avogadro (an open-source molecular builder and visualization tool. Version 1.90.0. http://avogadro.cc/). The force field MMF94 was set with number of steps 500, algorithm steepest descent and convergence of 10e-7 [28]. The structures of ligands were saved in Protein Data Bank (PDB) file format and used for docking study.

\section{Docking Between JAK2 and Ligands}

Discovery Studio visualizer and Chimera (UCSF, San Francisco, CA, USA) were chosen for visual inspection and preparations. PyRx software was employed as the virtual screening software. PyRx includes Auto Dock Vina with a Lamarckian genetic algorithm as a scoring algorithm. The ligand/protein simulated interactions of ruxolitinib (1), tofacitinib (2), rottlerin (5) and gandotinib (6) with JAK2 were determined using Auto Dock Vina (Molecular Graphics Lab, La Jolla, CA, USA) [28,29]. The docking was conducted with exhaustiveness of 8 and a grid box with the dimensions for center: $25 \times 25 \times 25 \AA$ and box center: center_x $=31.2851$, center_y $=0.4544$, center_z $=2.9915$ for 4AGC docking. PyMol v1.3 (Schrodinger, New York, NY, USA). Auto Dock Vina evaluated target conformation (biomacromolecule) as a rigid unit while ligands were conceded to be flexible and adoptable to the target. The software determined the lowest binding affinity by using different conformations of each ligand. AutoDock Vina searched for the lowest binding affinity conformations and determined 9 different conformations for each receptor and ligand complexes. Each receptor and ligands complex with the lowest binding energy docking poses were selected. The protein-ligand interactions were analyzed with LigPlot and Discovery Studio 4.5 (Dassault Systems BIOVIA, Discovery Studio Modelling Environment, Release 2017, San Diego, USA).

Docking simulations of target JAK2 with ruxolitinib (1), tofacitinib (2), rottlerin (5) and gandotinib (6) were performed in AutoDock Vina. (Molecular Graphics Lab, La Jolla, CA, USA). The AutoDock Vina software prepared the target in a rigid conformation while ligands were permitted to be flexible and malleable to the target. After the completion of docking, ligand conformations displaying greatest binding affinity and lowest docked energies to the target were selected. The hydrogen bonds, bond lengths and hydrophobic interactions between JAK2 and ligands ruxolitinib (1), tofacitinib (2), rottlerin (5) and gandotinib (6) were determined by using LigPlot (http://www.ebi.ac.uk/thornton-srv/software/ LIGPLOT/).

\section{Target-Ligand Complex Unbinding Simulations}

The MoMA-LigPath web server and Molecular Motion Algorithms (MoMA) were utilized to simulate ligand unbinding from the binding site to surface of the target. In addition, server discloses flexibility of protein side-chains, ligands and includes only statistical limitations. This process generates mechanistic data on the pathway of each ligand as it moves from the protein surface to the binding site or from binding site to surface of the protein. The program offers molecular interaction graphics, leading the ligands from surface of protein to the binding site. In this process, the program identifies certain residues that are crucial factors for

Cite this article: Jyothirmayi V, Anuradha V, Noboru M, Gollapudi R. Integrated in Silico Docking and MOMA Simulation Methods Reveal Rottlerin as a Potent Janus kinase 2 (JAK2) Inhibitor. Biomed J Sci\&Tech Res 11(1)-2018. BJSTR. MS.ID.002034. DOI: 10.26717/ BJSTR.2018.11.002034. ligand binding or driving ligands towards binding site, in spite of being away from binding site. The docked molecular complexes of JAK2 with ruxolitinib (1), tofacitinib (2), rottlerin (5) and gandotinib (6) that produced the lowest binding energies were selected for unbinding simulations by using MoMA LigPath [30,31].

\section{Results and Discussion}

Autonomous activation of the JAK/STAT pathway is an important factor in several pathologies. Genetic mutations targeting this signaling pathway are linked fundamentally with hematologic malignancies as well as diseases associated with uncharacteristic cytokine stimulation. The JAK/STAT pathway is frequently deregulated in malignant diseases caused by irregular immunological response. One of such disorder is myelofibrosis, a rare form of cancers of bone marrow where the bone marrow is replaced by scar tissue failing to produce healthy blood cells as a result of mutations. BCR-ABL1-negative myeloproliferative neoplasms initiate unusual activation of JAK2 caused by various mutations. The discovery of JAK2V617F as the driver mutation of majority of non-BCR-ABL1 myeloproliferative neoplasms encouraged the development of JAK inhibitors. Furthermore, search for JAK2 inhibitors continued following the discovery of JAK2V617F which revealed that other driver mutations like CALR and MPL also displayed tenacity in the activation of JAK2 [10].

The interpretation of the protein-ligand interactions plays an important role in structure-based drug discovery. When each ligand (1, 2, 5 and 6) was docked with JAK2, different binding energies were observed. Of these, known JAK inhibitors, tofacitinib (2) produced the lowest value $(-7.4 \mathrm{kcal} / \mathrm{mol})$, compared ruxolitinib (1) $(-8.7 \mathrm{kcal} / \mathrm{mol})$, and gandotinib (6) (-9.1 kcal/mol) whereas natural product rottlerin (5) generated the greatest binding energy $(-9.3) \mathrm{kcal} / \mathrm{mol})$ when docked with JAK2 receptor. These results suggest that rottlerin (5) might emerge as a promising candidate for the inhibition of JAK2 enzyme activity. The present docking study explored the interactions of ruxolitinib (1), tofacitinib (2), rottlerin (5) and gandotinib (6) with the receptor protein JAK2 and their binding patterns with JAK2 amino acid residues ruxolitinib (1), tofacitinib (2), rottlerin (5) and gandotinib (6) docked with the active site of JAK2.Each amino acid residue within a 4A0 distance from ligand was evaluated for the presence of any van der Waals forces or alkyl, and hydrogen bond interactions.

As a result, ruxolitinib (1), generated 11 van der Waals forces (Leu 24, Gly 25, Lys 26 , Gly 27, Lys 51, Glu 67, Val 80, Arg 149, Asn 150, Asp 163 and Phe 164) , 5 alkyl interactions (Val 32, Ala 49, Met 98, Ser 105 and Leu 152) and 1 hydrogen bond Asp 108 with JAK2; while tofacitinib (2) generated 7 van der Waals forces (Gly 25, Lys 26, Gly 27, Val 80, Arg 149, Gly 162 and Asp 163), 3 alkyl interactions (Leu 24, Val 32, Ala 49, Met 98 and Leu 152), with JAK2; gandotinib (6) generated 10 van der Waals forces (Gly 25, Lys 26, Gly 27, Ala 49, Pyr 100, Gly 104, Ser 105, Asp 108, Gly 162 and Asp 163), 5 alkyl interactions (Leu 24, Val 32, Val 80, Met 98, Arg 149 and Leu 152), 1hydrogen bond (Asn 150) with JAK2 residues whereas rottlerin (5) generated 8 van der Waals forces (Lys 26, Gly 25, Gly 27, Tyr 100, Leu 101, Gly 104, Asn 150 and Asp 163 ), 4 alkyl interactions (Leu 24, Val 32,Ala49 and Leu 152), 4 hydrogen bonds (Ser 105, Asp 
108, Arg 107 and Arg 149) with JAK2 residues. Collectively, these results suggested that rottlerin (5) might function as excellent JAK2 inhibitor since it produced the highest binding energy (-9.3 Kcal) (Figure 5).

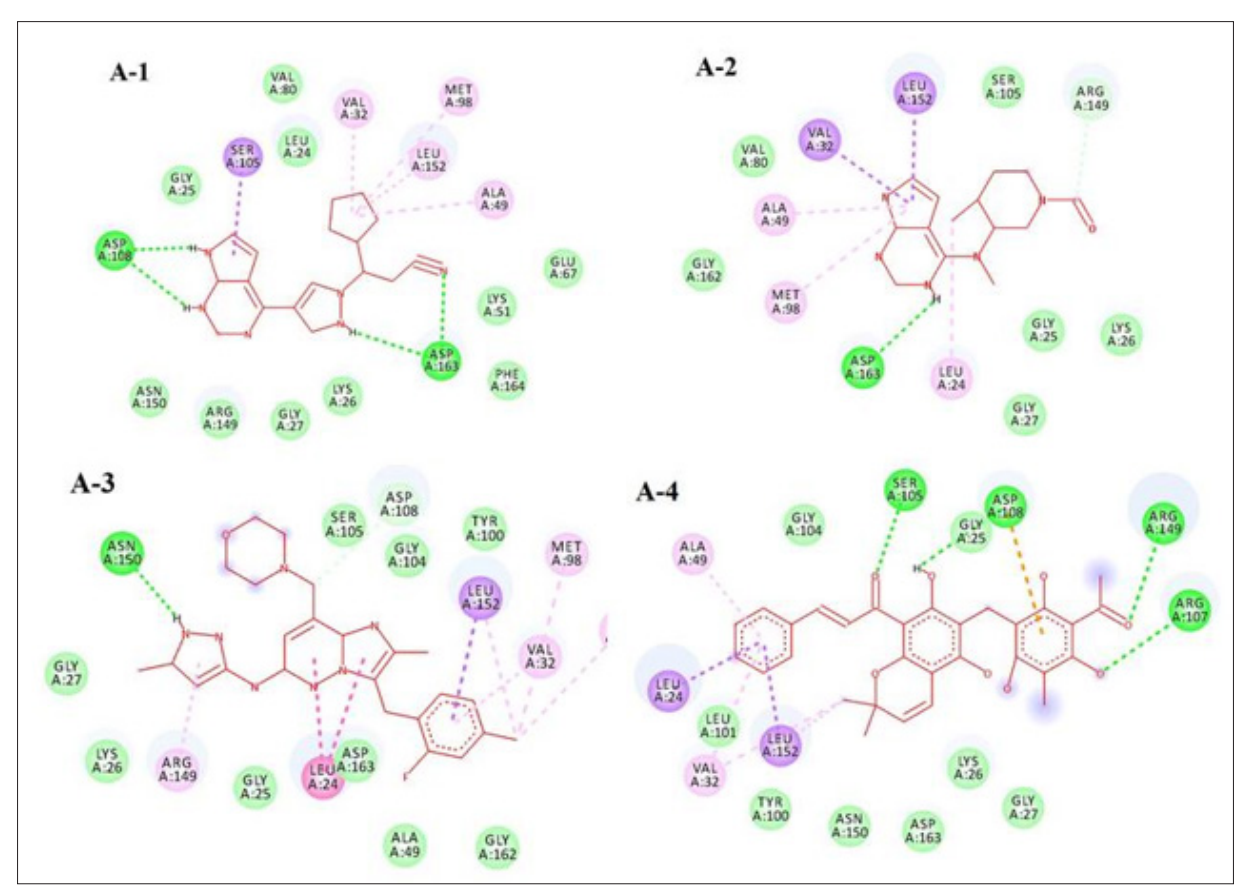

Figure 5: Ruxolitinib (1) (A-1), Tofacitinib (2) (A-2), Gandotinib (6) and rottlerin (5) binding interactions with JAK2.

\section{Unbinding Simulation of JAK2/Ruxolitinib Complex}

The unbinding simulation phases of ruxolitinib (1) with increasing number of molecular interactions while progressively approaching towards the binding site of JAK2 are shown in Figure
6 (B-1 to B-5). The docking phase displays molecular interactions of JAK2 with: ruxolitinib (1) bound on the exterior region of JAK2 cavity by hydrophobic interactions. A graphical representation of JAK2 in complex with ruxolitinib (1) was generated (Figure 6-B6).

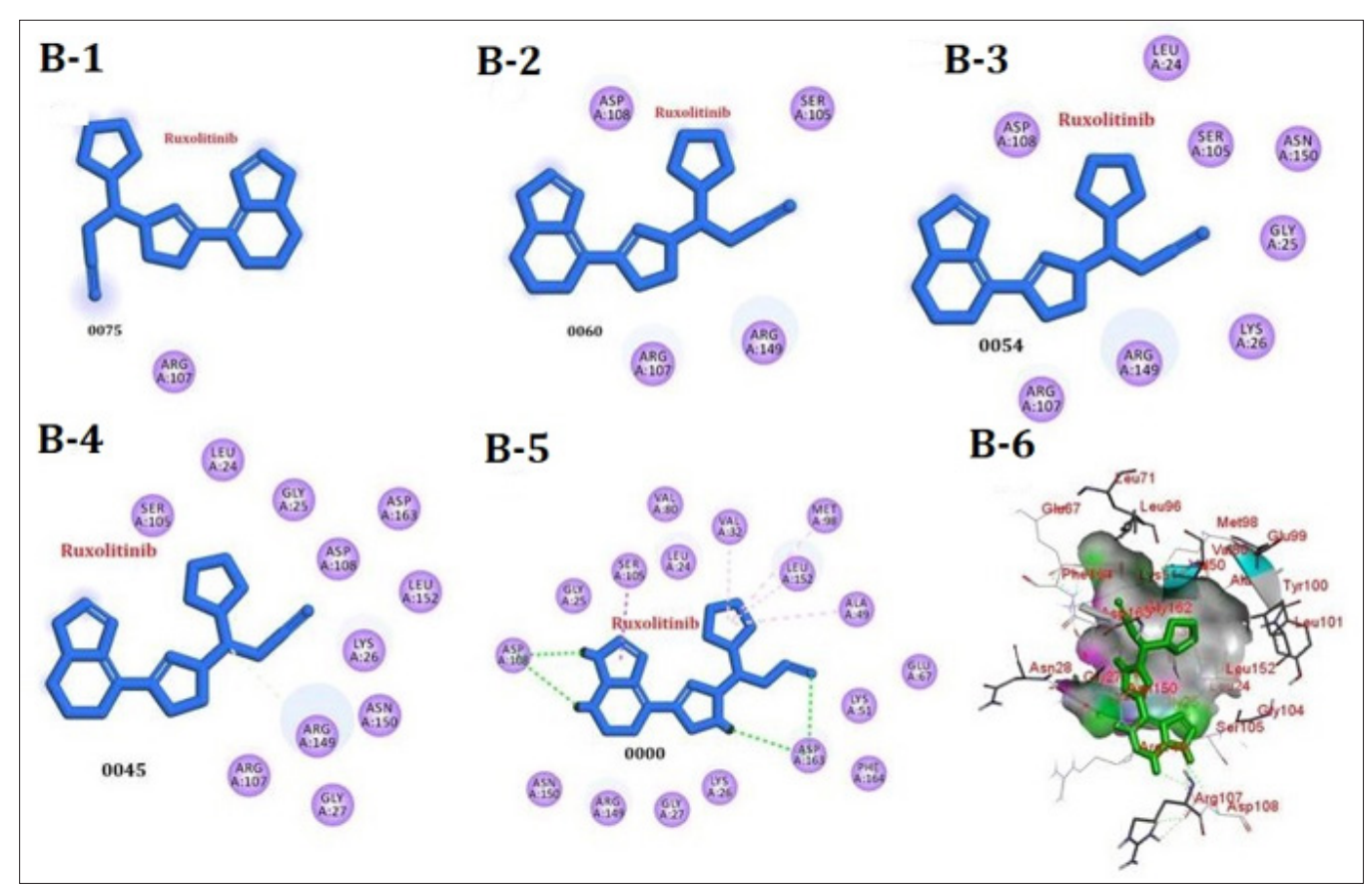

Figure 6: Ruxolitinib (1), binding to JAK2. Panels B-1 to B-5 show unbinding simulation phases of ruxolitinib: B-1 is the farthest from binding site, B-5 is the closest to binding site, and B-6 is binding site phase. Hydrogen bonds are shown as green-dashed lines with indicated bond length and the residues involved in hydrophobic interactions are shown as purple circles. 


\section{Unbinding Simulation of JAK2/Tofacitinib Complex}

The unbinding simulation phases of tofacitinib (2) with increasing number of molecular interactions while progressively approaching towards the binding site of JAK2 are shown in Figures
7-C1 to C5). The docking phase displays molecular interactions of JAK2 with: tofacitinib (2) bound on the exterior region of JAK2 cavity by hydrophobic interactions. A graphical representation of JAK2 in complex with tofacitinib (2) was generated (Figures 7-C6).

\section{C-1}
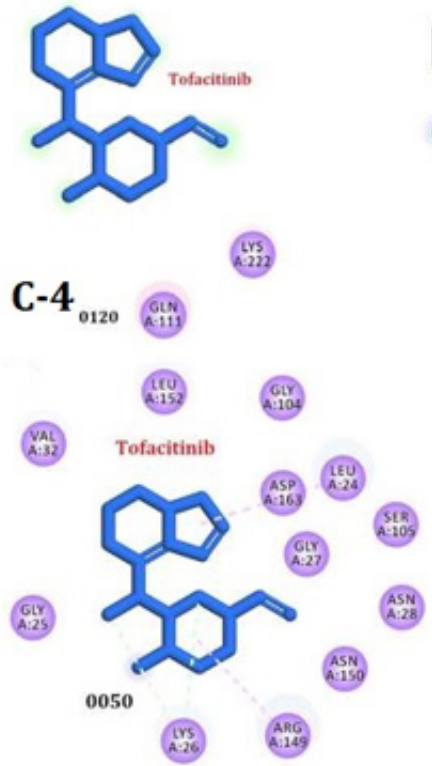

C-2

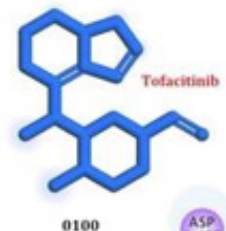

(1109)
C-5

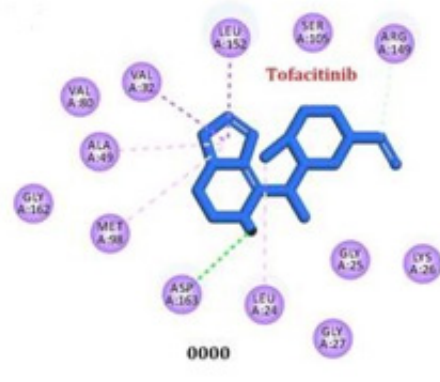

C-3

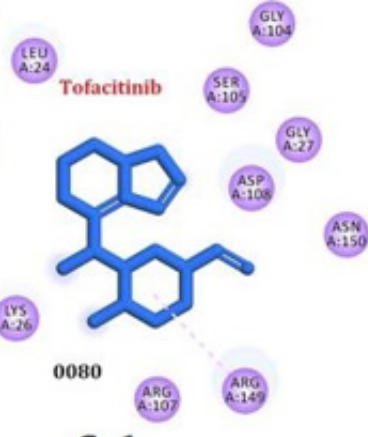

C-6

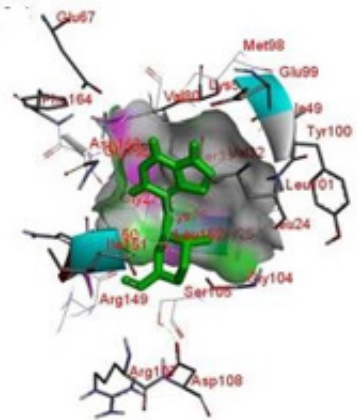

Figure 7: Tofacitinib (2) binding to JAK2. Panels C-1 to C-5 show unbinding simulation phases of tofacitinib (2): C-1 is the farthest from binding site, C-5 is the closest to binding site, and C-6 is binding site phase. Hydrogen bonds are shown as greendashed lines with indicated bond length and the residues involved in hydrophobic interactions are shown as purple circles.

\section{Unbinding Simulation of JAK2/Gandotinib Complex}

The unbinding simulation phases of gandotinib (3) with increasing number of molecular interactions while progressively approaching towards the binding site of JAK2 are shown in Figures
8-D1 to D5. The docking phase displays molecular interactions of JAK2 with: gandotinib (3), bound on the exterior region of JAK2 cavity by hydrophobic interactions. A graphical representation of JAK2 in complex with gandotinib (3) was generated (Figures 8-D6).

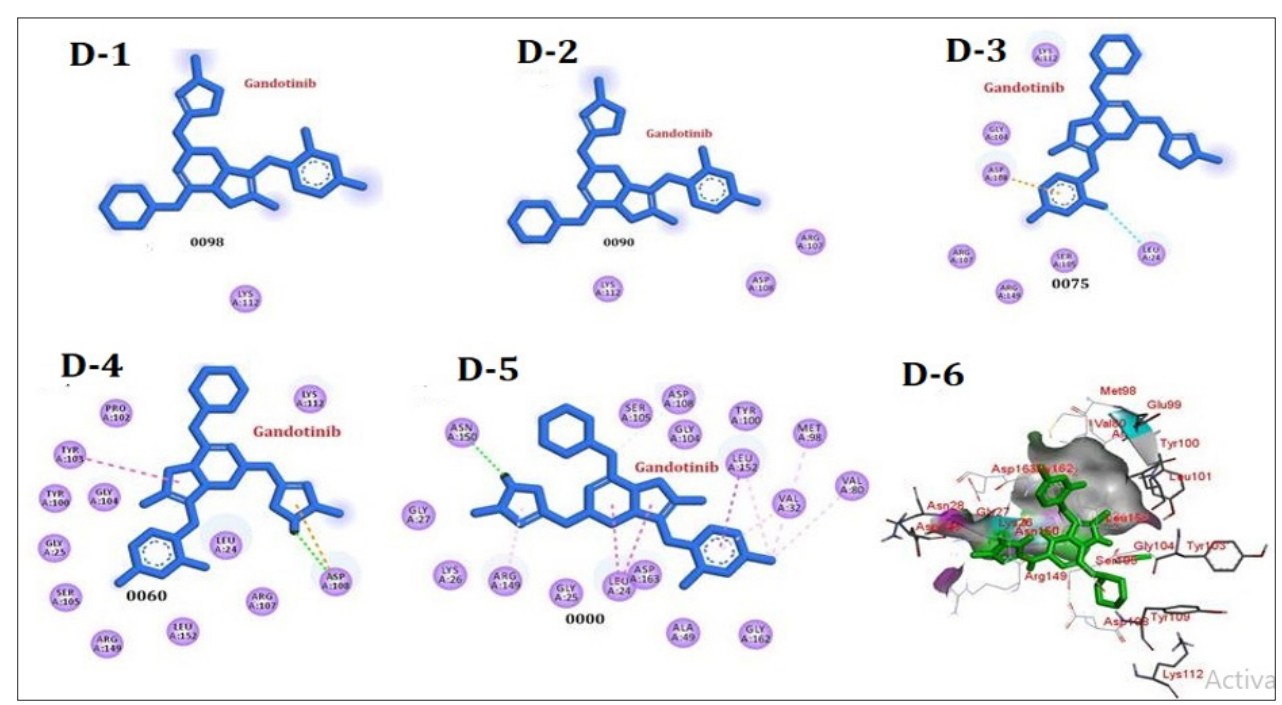

Figure 8: Gandotinib (6) binding to JAK2. Panels D-1 to D-5 show unbinding simulation phases of gandotinib (6): D-1 is the farthest from binding site, D-5 is the closest to binding site, and D-6 is binding site phase. Hydrogen bonds are shown as greendashed lines with indicated bond length and the residues involved in hydrophobic interactions are shown as purple circles. 


\section{Unbinding Simulation of JAK2/Rottlerin Complex}

The unbinding simulation phases of rottlerin (4) with increasing number of molecular interactions while progressively approaching towards the binding site of JAK2 are shown in Figures 9E-1 to E-5). The docking phase displays molecular interactions of JAK2 with: rottlerin (4) bound on the exterior region of JAK2 cavity by hydrophobic interactions. A graphical representation of JAK2 in complex with rottlerin (4) was generated (Figures 9-E6). In summary, rottlerin (5) docked to JAK2 with a better binding energy (-9.3K Cal), compared to other docked ligands: ruxolitinib (1) $(-8.7 \mathrm{kcal} / \mathrm{mol})$, tofacitinib (2), $(-7.4 \mathrm{kcal} / \mathrm{mol})$, and gandotinib (6) $(-9.1 \mathrm{kcal} / \mathrm{mol})$. In addition, MoMA server assisted unbinding simulation studies of JAK2/rottlerin, JAK2/ruxolitinib, JAK2/ tofacitinib and JAK2/gandotinib complexes suggested that rottlerin (5) took a longer time to unbind from JAK2 complex, compared to known JAK inhibitors, ruxolitinib (1), tofacitinib (2) and gandotinib (6). Based on this information, rottlerin (5) was selected for further investigations in order to evaluate its effectiveness as a competent inhibitor of JAK2 for the treatment of myelofibrosis and other disorders associated with JAKs expressions. A graphical representation of docked rottlerin (5), ruxolitinib (1), tofacitinib (2) (Blue) and gandotinib (6) in complex with JAK2 was generated (Figure 9). Rottlerin (5) and ATP occupying the active binding site of JAK2 is displayed (Figures 10 \& 11) [31].

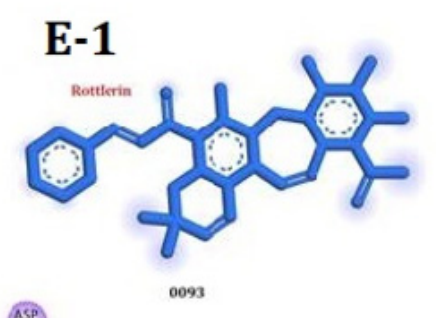

(ASP)

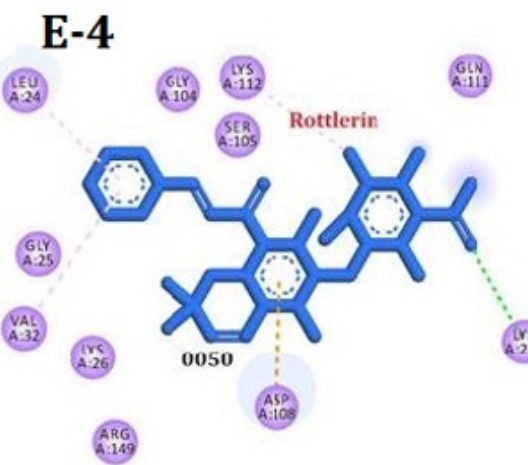

E-2

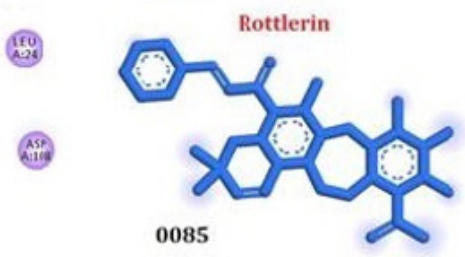

E5

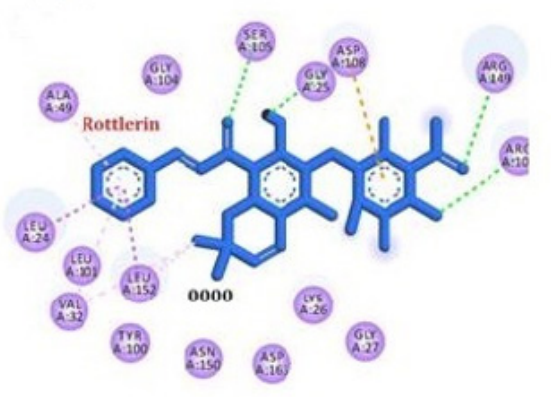

E-3

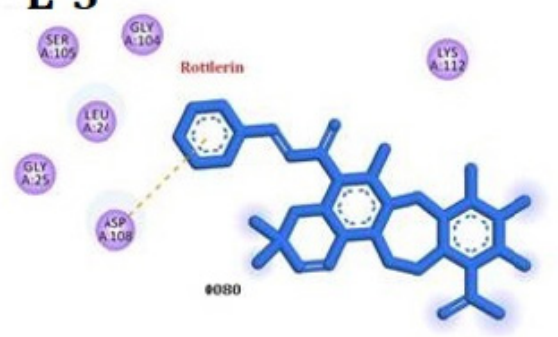

E-6

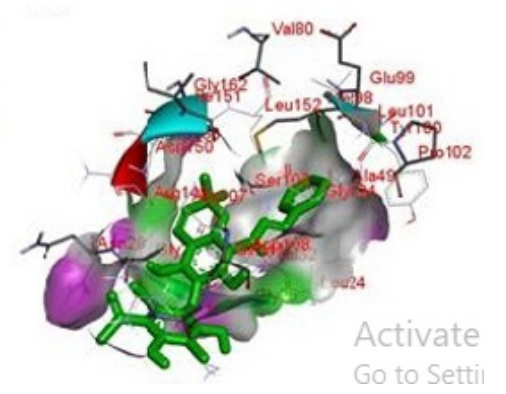

Figure 9: Rottlerin (5), binding to JAK2. Panels E-1 to E-5 show unbinding simulation phases of rottlerin (5): E-1 is the farthest from binding site, E-5 is the closest to binding site, and E-6 is binding site phase. Hydrogen bonds are shown as green-dashed lines with indicated bond length and the residues involved in hydrophobic interactions are shown as purple circles.

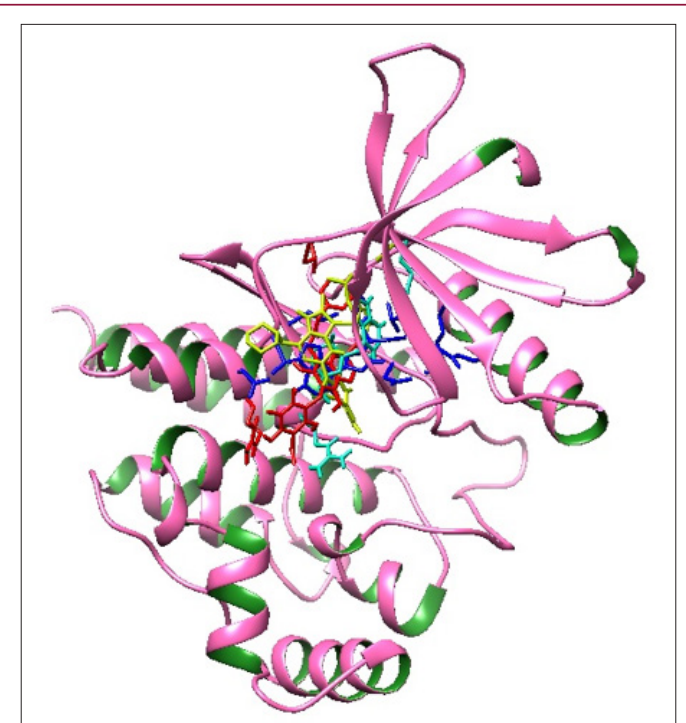

Figure 10: Ruxolitinib (1) (Red), tofacitinib (2) (Blue), gandotinib (6) (Green) and rottlerin (5) (Green) docked on JAK2. 


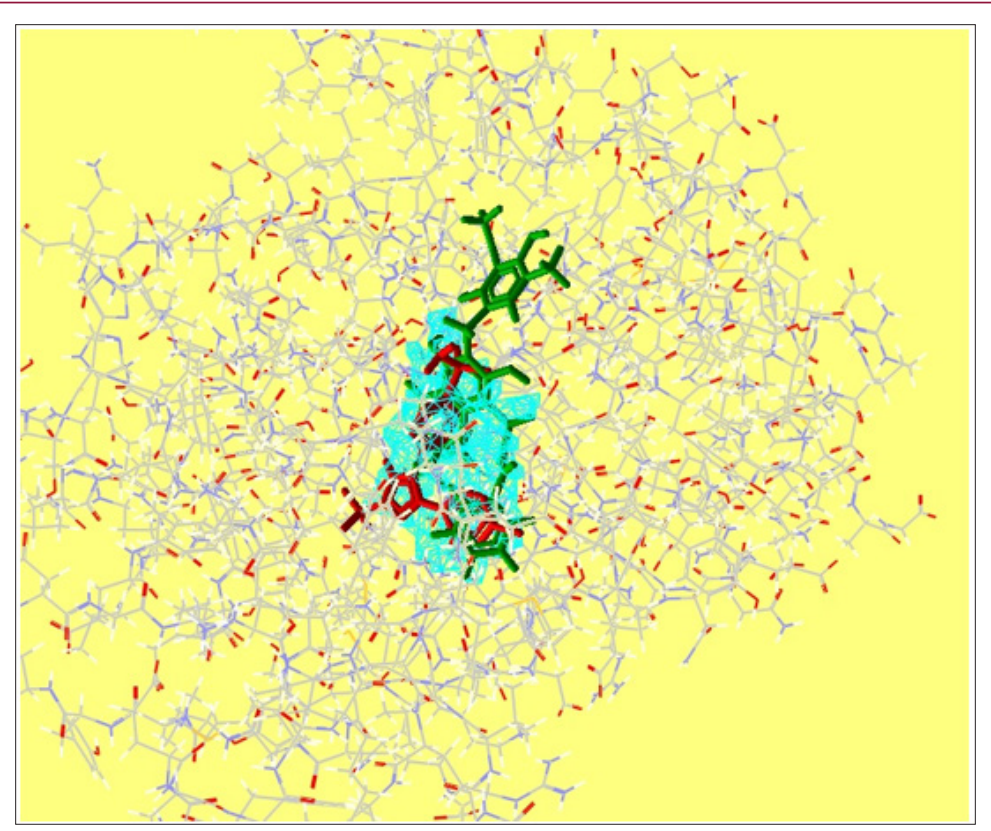

Figure 11: Rottlerin (5) (Green) and ATP (RED) docked on JAK2 active cavity (Cayman Blue).

\section{Conclusion}

Myelofibrosis is a cluster of rare bone marrow cancers also known as chronic leukemia that belongs to certain type of blood disorders-myeloproliferative ailments. In case of mylofibrosis one blood stem cell inherits the capacity to reproduce without any regulation and thus generating large numbers of immature blood cells leaving little room for healthy cells. The discovery of JAK2 mutations initiated the discovery of a targeted therapy for myelofibrosis. BCR-ABL1-negative myeloproliferative neoplasms are associated with abnormal JAK2 activation. Most often, the JAK/ STAT pathway is disrupted in malignant disorders with abnormal immunological responses. The discovery of JAK2V617F as a driver mutation of majority of non-BCR-ABL1 myeloproliferative neoplasms in 2005 led to the development of JAK inhibitors. At present, ruxolitinib (1) is the sole FDA approved medication available for the treatment of myelofibrosis.

Our present in silico docking study suggested that rottlerin (5), a natural product displayed strong interactions with JAK2 through hydrogen bonds, van der Waals and alkyl forces with binding energy $-9.3 \mathrm{kcal} / \mathrm{mol}$. Additionally, unbinding simulation studies on: ruxolitinib (1), tofacitinib (2), gandotinib (6) and rottlerin (5) in complex with JAK2 revealed that rottlerin (5) took a longer time to unbind from JAK2, compared to ruxolitinib (1), tofacitinib (2) and gandotinib (6). In addition, these data supported our in silico observations for rottlerin (5). The current docking investigations strongly support future research to evaluate the potential of rottlerin (5) as JAK2 inhibitors in the treatment of myeloproliferative neoplasms. In summary, these results suggested that rottlerin (1) might function as better JAK2 inhibitor and should be explored further as a potential solution for the treatment of myelofibrosis.

\section{References}

1. Bole Feysot C, Gofin V, Edery M, Binart N, Kelly PA (1998) Prolactin (PRL) and its receptor: actions, signal transduction pathways and phenotypes observed in PRL receptor knockout mice. Endocrine Reviews 19(3): 225-268.

2. Brooks AJ, Dai W, O Mara ML, Abankwa D, Chhabra Y, et al. (2014) Mechanism of activation of protein kinase JAK2 by the growth hormone receptor". Science 344 (6185): 1249783.

3. Yamaoka K, Saharinen P, Pesu M, Holt VE, Silvennoinen O, et al. (2004) The janus kinases (Jaks). Genome Biol 5(12): 253

4. Marotta LLC, Almendro V, Marusyk A, Shipitsin M, Schemme J, et al. (2011) The JAK2/STAT3 signaling pathway is required for growth of CD44+CD24-stem cell-like breast cancer cells in human tumors. J Clin Invest 121(7): 2723-2735.

5. Levine RL, Wadleigh M, Cools J, Ebert BL, Wernig G, et al. (2005) Activating mutation in the tyrosine kinase JAK2 in polycythemia Vera, essential thrombocythemia, and myeloid metaplasia with myelofibrosis. Cancer Cell 7(4): 387-397.

6. James C, Ugo V, Le Couédic JP, Staerk J, Delhommeau F, et al. (2005) A unique clonal JAK2 mutation leading to constitutive signaling causes polycythaemia Vera. Nature 434(7037): 1144-1148.

7. Baxter EJ, Scott LM, Campbell PJ, East C, Fourouclas N, et al. (2005) Acquired mutation of the tyrosine kinase JAK2 in human myeloproliferative disorders. Lancet 365(9464): 1054-1061.

8. Verma A, Kambhampati S, Parmar S, Platanias LC (2003) Jak family of kinases in cancer. Cancer Metastasis Rev. 22(4):423-434.

9. Santos FPS, Kantarjian HM, Jain N, Manshouri, Thomas DA, et al. (2010) Phase 2 study of CEP-701, an orally available JAK2 inhibitor, in patients with primary or post-polycythemia Vera/essential thrombocythemia myelofibrosis. Blood 115(6): 1131-1136.

10. Vainchenker W, Leroy E, Gilles L, Marty C, Plo I, et al. (2018) JAK inhibitors for the treatment of myeloproliferative neoplasms and other disorders. F1000Res 7: 82.

11. Yao T, Xie J, Liu X, Cheng J, Zhu C, et al. (2017) Integration of pharmacophore mapping and molecular docking in sequential virtual 
screening: towards the discovery of novel JAK2 inhibitors. RSC Advances 7(17): 10353-10360.

12. Lin Y, Wang F, Zhang GL (2014) Natural products and their derivatives regulating te janus kinase/signal transducer and activator of transcription pathway. J Asian Nat Prod Res 16(7): 800-812.

13. Lim H, Park H, Kim HP (2011) Effects of flavonoids on matrix metalloproteinase-13 expression of interleukin-1 $\beta$-treated articular chondrocytes and their cellular mechanisms: inhibition of c-Fos/AP-1 and JAK/STAT signaling pathways. J Pharmacol Sci 116(2): 221-231.

14. Maioli E, TorricelliC, ValacchiG (2012) Rottlerin and cancer: novel mechanisms. Sci World J: 350826.

15. Bain J, Plater L, Elliott M, Shpiro N, Hastie CJ, et al. (2007). The selectivity of protein kinase inhibitors: a further update, Biochem J 408(3): 297 315.

16. Tapia JA, Jensen RT, Garcia Marin LJ (2006) Rottlerin inhibits stimulated enzymatic secretion and several intracellular signaling transduction pathways in pancreatic acinar cells by a non-PKC- $\delta$-dependent mechanism. Biochimica et Biophysica Acta 1763(1): 25-38.

17. Su J, Wang L, Yin X, Zhao Z, Yingying Hou Y, et al. (2016) Rottlerin exhibits anti-cancer effect through inactivation of $\mathrm{S}$ phase kinase-associated protein 2 in pancreatic cancer cells. Am J Cancer Res 6(10): 2178-2191.

18. Juneja M, Kobelt D, Walther Q, Voss C, Smith J, et al. (2017) Statin and rottlerin small-molecule inhibitors restrict colon cancer progression and metastasis via MACC1. PLoS Biol 15(6): e2000784.

19. Chothia C, Lesk AM (1986) The relation between the divergence of sequence and structure in proteins. EMBO J 5(4): 823-826.

20. Kaczanowski S, Zielenkiewicz P (2010) Why similar protein sequences encode similar three-dimensional structures? Theor Chem Acc 125(36): 643-650.

21. Bordoli L, Kiefer F, Arnold K, Benkert P, Battey J, et al. (2008) Protein structure homology modelling using SWISS-MODEL workspace. Nature Protocols 4(1): 1-13.

ISSN: 2574-1241

DOI: 10.26717/BJSTR.2018.11.002034

Rao Gollapudi. Biomed J Sci \& Tech Res

(C) (i) This work is licensed under Creative

Submission Link: https://biomedres.us/submit-manuscript.php
22. Schwede T, Kopp J, Guex N, Peitsch MC (2003) SWISS-MODEL: an automated protein homology-modeling serve. Nucleic Acids Research 31(13): 3381-3385.

23. Biasini M, Bienert S, Waterhouse A, Arnold K, Studer G, et al. (2014) SWISS-MODEL: modelling protein tertiary and quaternary structure using evolutionary information. Nucleic Acids Research 42(W1): 195201.

24. Read RJ, Adams PD, Arendall III WB, Brunger AT, Emsley P, et al. (2011). A New generation of crystallographic validation tools for the protein data bank. Structure 19(10): 1395-1412.

25. Savarino A (2007) In Silico docking of HIV-1 integrase inhibitors reveals a novel drug type acting on an enzyme/DNA reaction intermediate. Retrovirology 4(21): 1-15.

26. Nousheen L, Akkiraju PC, Enaganti S (2014) Molecular docking mutational studies on human surfactant protein-D. World J Pharmaceut Res, 3(7): 1140-1148.

27. Jin H, Zhou Z, Wang D, Guan S and Han W (2015) Molecular dynamics simulations of acylpeptide hydrolase bound to chlopyrifosmethyl oxon and dichlorvos. Inter J Mol Sci 16(3): 6217-6234.

28. Hanwell MD, Curtis DE, Lonie DC, Vandermeersch T, Zurek E, et al. (2012) Avogadro: An advanced semantic chemical editor, visualization, and analysis platform. J Cheminform 4(1): 17.

29. Dallakyan S, Oleson A (2015) Small-molecule library screening by docking with PyRx Methods. Mol Biol 1263: 243-250.

30. Devaurs D, Bouard L, Vaisset M, Zanon C, Al Bluwi I, et al. (2013) MoMALigPath: a web server to simulate protein-ligand unbinding. Nucleic Acids Research 41(W1): W297-W302.

31. Cortes J, Le DT, Iehl R, Simeon T (2010) Simulating ligand-induced conformational changes in proteins using a mechanical disassembly method. Physical Chem Chem Phys 12(29): 8268-8276.

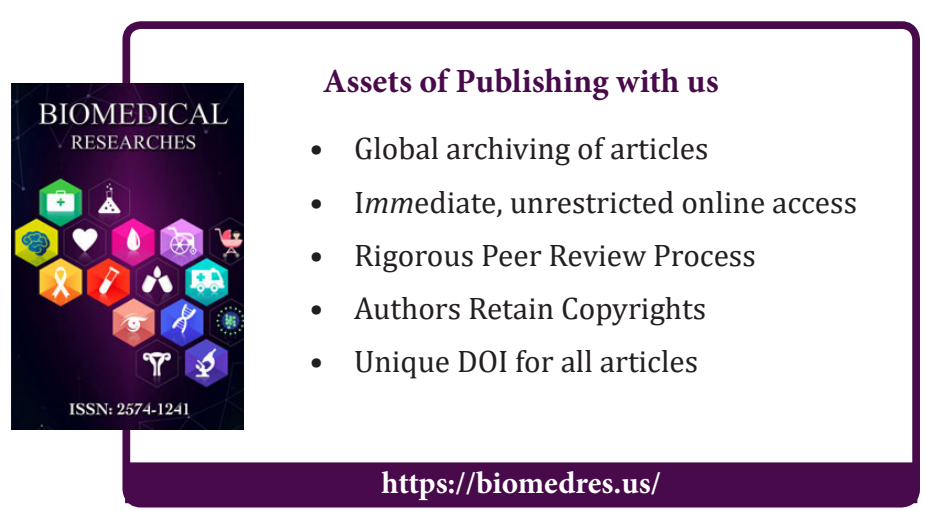

